\title{
Research on Attitude Control Method of Agile Satellite Based on Variable Structure Control Algorithm
}

\author{
Jian Liang*, Xin Zhao, Yang Zhao, Hefen Zhang, Yawen Cai, Yang Sun and \\ He Liu
}

Beijing Institute of Spacecraft System Engineering, No. 104,Youyi Road, Haidian District, Beijing, China

${ }^{*}$ Corresponding author

Keywords: Agile satellites, Attitude maneuver, SGCMG, Variable structure.

\begin{abstract}
With the improvement of remote sensing satellite observation capability, agile satellites with rapid mobility have attracted increasing attention. In order to realize the high pre-cision earth observation and stereo imaging in the same orbit, the earth observation satellite needs to have the ability of fast attitude maneuver and stabilization. The satellite needs to have the ability of fast attitude maneuver to track the target. In order to realize the fast attitude maneuver of agile satellite, the Pentagon configuration composed of six SGCMGs is used as the attitude control actuator. In order to shorten the time required for attitude maneuver of satellite, the angular trajectory of attitude maneuver around Euler axis is planned, and the error quaternion between the actual attitude and the planned attitude of satellite is calculated. Variable structure control law is designed with error angular velocity. The simulation results show that the control law designed in this paper can effectively complete the attitude maneuver around the Euler axis and has strong robustness.
\end{abstract}

\section{Introduction}

In recent years, the agile satellites have got much attention. Compared to conventional satellites, agile satellites take less time to retarget and have better performance in the effectiveness of utilization. Agile satellites can be of great essence in earth observation, space surveillance and satellite monitoring, etc. The rapid maneuver ability of agile satellites advances the requirements of attitude control system. However, the typical momentum exchange actuators, such as reaction wheels, may not content these requirements. Single gimbal control moment gyroscopes (SGCMG) can produce much larger control torque than reaction wheels do. Besides, the energy consumption of a SGCMG is low. These advantages enable SGCMGs to be ideal actuators for agile satellites.

Focusing on this issue, a number of control methods have been proposed. In the work of [1] and [2], a nondiagonal weighting matrix was utilized and CMG steering logic based on the simple minimum two-norm, pseudoinverse solution was proposed. A hybrid steering logic was presented in the work of [3], analysis and simulations are presented to demonstrate the performance of the hybrid steering logic in the work of [4]. A variable structure control in natural frame for a three-phase girdconnected voltage-source inverter with LCL filter was presented in [5].The proposed control method is based on modifying the converter model in natural reference frame, preserving the low-frequency state-space variables dynamics. Some problems about the attitude maneuver control are discussed in this paper. The attitude dynamic model of a flexible satellite with SGCMGs and the kinematical model in quaternions are established, these lay the foundation for the analysis and design of attitude control system. In order to accomplish the attitude maneuver control mission, an angular path for maneuvering is planned. An attitude tracking model based on error quaternions is established. After that a variable structure control algorithm are designed. Simulations for the algorithms suggest that the control law perform well, while the variable structure algorithm is not sensitive to the parameter uncertainty and external disturbance. 


\section{Mathematical Model of Agile Satellite}

The dynamical equation of rigid satellite can be expressed as follows:

$$
\boldsymbol{J} \dot{\boldsymbol{\omega}}+\dot{\boldsymbol{h}}+\boldsymbol{\omega} \times(\boldsymbol{J} \boldsymbol{\omega}+\boldsymbol{h})=\boldsymbol{T}_{d}
$$

where $\boldsymbol{J}$ is the inertia matrix, $\boldsymbol{\omega}$ is the angular velocity in inertial system, $\boldsymbol{h}$ is the angular momentum and $\boldsymbol{T}_{d}$ is the disturbing torque. If the SGCMG system output control torque is expressed as $\boldsymbol{u}=-\dot{\boldsymbol{h}}-\boldsymbol{\omega} \times \boldsymbol{h}$, then the dynamical equation of rigid satellite can be redefined as:

$$
J \dot{\omega}=T_{d}-\omega \times J \omega+u
$$

Error quaternion is used to represent the attitude kinematics model of the satellite. Firstly, the attitude quaternion of the satellite relative to the orbit coordinate system is defined as:

$$
\boldsymbol{q}=\left[\begin{array}{llll}
q_{0} & q_{1} & q_{2} & q_{3}
\end{array}\right]^{T}
$$

where $q_{0}=\cos (\phi / 2), q_{13}=\left[\begin{array}{lll}q_{1} & q_{2} & q_{3}\end{array}\right]^{T}=\boldsymbol{n} \sin (\phi / 2), \boldsymbol{n}$ is the unit vector in the direction of Euler axis, $\phi$ is the angle of rotation around the Euler axis. And the quaternion satisfies the constraint condition of $\boldsymbol{q}^{T} \boldsymbol{q}=1$, in this paper we assume that $q_{0}>0$.

Assuming that the planning attitude quaternion is $\boldsymbol{q}_{s}$ and the error quaternion between $\boldsymbol{q}$ and $\boldsymbol{q}_{s}$ is $\boldsymbol{q}_{e}$ can be presented as:

$$
\boldsymbol{q}_{e}=\boldsymbol{q}_{s}^{-1} \otimes \boldsymbol{q}
$$

where $\boldsymbol{q}_{s}^{-1}=\left[\begin{array}{llll}q_{0} & -q_{1} & -q_{2} & -q_{3}\end{array}\right]^{T}$, $\otimes$ means multiplication of quaternion. The kinematics equation based on attitude quaternion is:

$$
\dot{\boldsymbol{q}}_{e}=\frac{1}{2} A\left(\boldsymbol{q}_{e}\right) \cdot\left(\omega_{r}-\omega_{s}\right)
$$

where $\omega_{r}$ is the angular velocity of the body coordinate system relative to the orbital coordinate

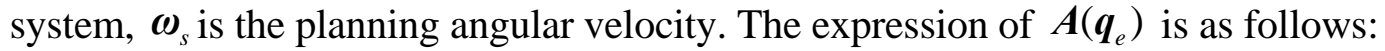

$$
A\left(\boldsymbol{q}_{e}\right)=\left[\begin{array}{ccc}
-q_{e 1} & -q_{e 2} & -q_{e 3} \\
q_{e 0} & -q_{e 3} & q_{e 2} \\
q_{e 3} & q_{e 0} & -q_{e 1} \\
-q_{e 2} & q_{e 1} & q_{e 0}
\end{array}\right]
$$

The angular velocity of the body coordinate system relative to the inertial system can be expressed as:

$$
\omega=\omega_{r}+T_{b o}(q) \omega_{o}
$$

where $\omega_{o}=\left[\begin{array}{lll}0 & -\omega_{0} & 0\end{array}\right]^{T}$ is orbit angular velocity and the transformation matrix from orbital coordinate system to body coordinate system can be presented as:

$$
\boldsymbol{T}_{\text {bo }}(\boldsymbol{q})=\left[\begin{array}{lll}
2\left(q_{0}^{2}+q_{1}^{2}\right)-1 & 2\left(q_{1} q_{2}+q_{0} q_{3}\right) & 2\left(q_{1} q_{3}-q_{0} q_{2}\right) \\
2\left(q_{1} q_{2}-q_{0} q_{3}\right) & 2\left(q_{0}^{2}+q_{2}^{2}\right)-1 & 2\left(q_{2} q_{3}+q_{0} q_{1}\right) \\
2\left(q_{1} q_{3}+q_{0} q_{2}\right) & 2\left(q_{2} q_{3}-q_{0} q_{1}\right) & 2\left(q_{0}^{2}+q_{3}^{2}\right)-1
\end{array}\right]
$$




\section{The Steering Law Design for SGCMG System}

For a single SGCMG, the output torque is located on the angular momentum surface perpendicular to the frame axis, and the direction of the output torque is always orthogonal to the direction of the angular momentum. As shown in the figure 1, the direction of the output torque of the SGCMG is

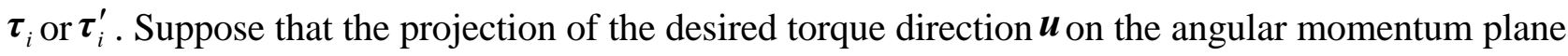
is $\boldsymbol{\tau}_{d i}$, when the projection of the angular momentum vector $\boldsymbol{h}_{i}$ and the expected torque $\boldsymbol{\tau}_{d i}$ are orthogonal to each other, the output torque produced by the change of the angular momentum of SGCMG is the same as the projection direction of the expected torque. At this time, the torque output capability of SGCMG in the desired direction reaches the maximum. For SGCMG systems, the following torque output performance specifications can be defined:

$$
S=\sum_{i=1}^{n} \sin ^{2} \theta_{i}
$$

where $\theta_{i}$ is the angle between the ith SGCMG angular momentum vector $\boldsymbol{h}_{i}$ and the projection of expected torque $\boldsymbol{\tau}_{d i}$, and is the number of SGCMGs in the system, $n$ is the number of SGCMGs in the system. The bigger the $S$ is, the bigger the torque output capability in the desired direction is. $S=0$ shows that the angular momentum direction of each SGCMG of the system is the same or opposite as the projection direction of the expected torque. At this time, the system can't produce the output torque in the desired direction, and the system falls into a singular state. Compared with the singularity measure $D$, the torque output performance index $S$ can better reflect the torque output performance of the system in the desired direction.

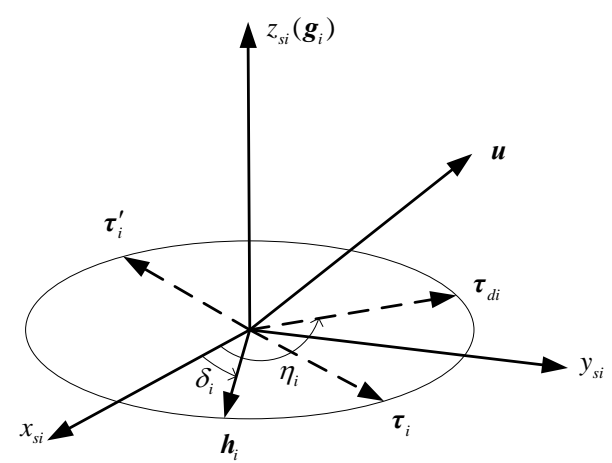

Figure 1. The schematic diagram of SGCMG torque output.

The rotation speed of the gimbal can be decomposed into two parts which can be written as:

$$
\dot{\boldsymbol{\delta}}=\dot{\boldsymbol{\delta}}_{T}+\dot{\boldsymbol{\delta}}_{N}
$$

where $\dot{\boldsymbol{\delta}}_{T}$ is the rotational speed with torque output and $\dot{\boldsymbol{\delta}}_{N}$ is the rotational speed without torque output. These two variables satisfy the following equation.

$$
\begin{aligned}
& \boldsymbol{C} \dot{\boldsymbol{\delta}}_{T}=-\boldsymbol{T}_{c} / \mathrm{h} \\
& \boldsymbol{C} \dot{\boldsymbol{\delta}}_{N}=0
\end{aligned}
$$

The solution of this equation is:

$$
\dot{\boldsymbol{\delta}}_{N}=\left[\boldsymbol{E}_{n}-\boldsymbol{C}^{T}\left(\boldsymbol{C} \boldsymbol{C}^{T}\right)^{-1} \boldsymbol{C}\right] \boldsymbol{v}
$$

where $\boldsymbol{v}$ is n dimensional vector and $\boldsymbol{E}_{n}$ is n dimensional unit matrix. In the process of gimbal rotation, the performance index of torque output $S$ will change. The expression of $S$ changing with the frame rotation angle can be expressed as follows: 


$$
\dot{S}=\frac{\partial S}{\partial \boldsymbol{\delta}^{T}} \dot{\boldsymbol{\delta}}=\frac{\partial S}{\partial \boldsymbol{\delta}^{T}} \dot{\boldsymbol{\delta}}_{T}+\frac{\partial S}{\partial \boldsymbol{\delta}^{T}} \dot{\boldsymbol{\delta}}_{N}
$$

In this equation, the expressions of some variables are as follows:

$$
\begin{aligned}
\frac{\partial S}{\partial \boldsymbol{\delta}^{T}} & =\left(\frac{\partial S}{\partial \delta_{1}}, \frac{\partial S}{\partial \delta_{2}}, \cdots, \frac{\partial S}{\partial \delta_{n}}\right) \\
\frac{\partial S}{\partial \delta_{i}} & =2 \sin \theta_{i} \cdot \cos \theta_{i}
\end{aligned}
$$

We make a definition that $R=\frac{\partial S}{\partial \boldsymbol{\delta}^{T}} \dot{\boldsymbol{\delta}}_{N}$ and combine (12) with this definition, the expression of R can be written as:

$$
R=\frac{\partial S}{\partial \boldsymbol{\delta}^{T}}\left[\boldsymbol{E}_{n}-\boldsymbol{C}^{T}\left(\boldsymbol{C} \boldsymbol{C}^{T}\right)^{-1} \boldsymbol{C}\right] \boldsymbol{v}=\boldsymbol{V}^{T} \boldsymbol{v}
$$

where $\boldsymbol{V}^{T}=\frac{\partial S}{\partial \boldsymbol{\delta}^{T}}\left[\boldsymbol{E}_{n}-\boldsymbol{C}^{T}\left(\boldsymbol{C} \boldsymbol{C}^{T}\right)^{-1} \boldsymbol{C}\right]$, when the undetermined vector $\boldsymbol{v}$ is parallel to the vector $\boldsymbol{V}$, $R$ has a positive maximum value. Under this assumption, the rotational speed without torque output can be expressed as:

$$
\begin{aligned}
\dot{\boldsymbol{\delta}}_{N} & =\lambda\left[\boldsymbol{E}_{n}-\boldsymbol{C}^{T}\left(\boldsymbol{C} \boldsymbol{C}^{T}\right)^{-1} \boldsymbol{C}\right]\left[\boldsymbol{E}_{n}-\boldsymbol{C}^{T}\left(\boldsymbol{C} \boldsymbol{C}^{T}\right)^{-1} \boldsymbol{C}\right] \frac{\partial S}{\partial \boldsymbol{\delta}} \\
& =\lambda\left[\boldsymbol{E}_{n}-\boldsymbol{C}^{T}\left(\boldsymbol{C} \boldsymbol{C}^{T}\right)^{-1} \boldsymbol{C}\right] \frac{\partial S}{\partial \boldsymbol{\delta}}
\end{aligned}
$$

So the steering law based on the performance index of torque output is:

$$
\dot{\boldsymbol{\delta}}=-\boldsymbol{C}^{T}\left(\boldsymbol{C} \boldsymbol{C}^{T}\right)^{-1} \boldsymbol{T}_{c} / h+\lambda\left[\boldsymbol{E}_{n}-\boldsymbol{C}^{T}\left(\boldsymbol{C} \boldsymbol{C}^{T}\right)^{-1} \boldsymbol{C}\right] \frac{\partial S}{\partial \boldsymbol{\delta}}
$$

The magnitude of zero motion coefficient $\lambda$ reflects the degree of the control law's ability to maintain torque output in the desired direction through zero motion. The larger the value $\lambda$ is, the stronger the steering law's ability to output torque in the desired direction. The system can't acquire larger torque output capability in the desired direction, so the coefficients $\lambda$ need to be selected properly. When the output performance index of torque $S$ is large, the output torque capability of the system in the desired direction is larger, and the zero motion coefficient $\lambda$ can be smaller, when the output torque is small, the output torque capability in the desired direction is smaller, and the zero motion coefficient should be larger. Therefore, the coefficient $\lambda$ can be written as follows:

$$
\lambda=\lambda_{0} \exp (-b \cdot S)
$$

\section{The Variable Structure Controller Design}

\section{The Sliding Mode Design}

The sliding mode can be designed as followed:

$$
\boldsymbol{s}=\left(\boldsymbol{\omega}_{r}-\boldsymbol{\omega}_{s}\right)+\boldsymbol{G} \boldsymbol{q}_{e 13}
$$

where $\mathrm{G}$ is the definite diagonal matrix and $\boldsymbol{q}_{e 13}=\left[\begin{array}{lll}q_{e 1} & q_{e 2} & q_{e 3}\end{array}\right]^{T}$, when the system working on the sliding mode, $\boldsymbol{s}=\boldsymbol{0}$, we take the Lyapunov function as:

$$
V=\frac{1}{2} \boldsymbol{q}_{e 13}^{T} \boldsymbol{G} \boldsymbol{q}_{e 13}
$$


where $V \geq 0$, the derivative of the Lyapunov function is:

$$
\dot{V}=-\boldsymbol{q}_{e 13}^{T} \boldsymbol{G} \dot{\boldsymbol{q}}_{e 13}=-\frac{1}{2} q_{e 0}\left(\boldsymbol{G} \boldsymbol{q}_{e 13}\right)^{T} \boldsymbol{G} \boldsymbol{q}_{e 13} \leq 0
$$

If and only if $\boldsymbol{q}_{e 13}=\boldsymbol{0}$, then $\dot{V}=0$. Therefore, the system is asymptotically stable in sliding mode.

\section{The Variable Structure Control Law Design}

The derivative of the sliding mode can be expressed as:

$$
\dot{\boldsymbol{s}}=\left(\dot{\boldsymbol{\omega}}_{r}-\dot{\boldsymbol{\omega}}_{s}\right)+\boldsymbol{G} \dot{\boldsymbol{q}}_{e 13}
$$

In order to satisfy the reachability of the sliding mode, we choose the reaching law as follow:

$$
\dot{\boldsymbol{s}}=-\boldsymbol{K} \boldsymbol{s}-\boldsymbol{W} \operatorname{sgn}(\boldsymbol{s})
$$

where $\boldsymbol{K}$ and $\boldsymbol{W}$ are all definite diagonal matrix, then we can get:

$$
\dot{\boldsymbol{\omega}}=-\boldsymbol{K} \boldsymbol{s}-\boldsymbol{W} \operatorname{sgn}(\boldsymbol{s})-\boldsymbol{G} \dot{\boldsymbol{q}}_{e 13}+\dot{\boldsymbol{\omega}}_{s}+\dot{\boldsymbol{T}}_{b o}(\boldsymbol{q}) \boldsymbol{\omega}_{0}
$$

When Ignoring disturbance torque, the expression can be written as follow:

$$
\begin{aligned}
\boldsymbol{u} & =\boldsymbol{J} \dot{\boldsymbol{\omega}}+\boldsymbol{\omega} \times \boldsymbol{J} \boldsymbol{\omega} \\
& =-\boldsymbol{J}\left[\boldsymbol{K} \boldsymbol{s}+\boldsymbol{W} \operatorname{sgn}(\boldsymbol{s})+\boldsymbol{G} \dot{\boldsymbol{q}}_{e 13}-\dot{\boldsymbol{\omega}}_{s}-\dot{\boldsymbol{T}}_{b o}(\boldsymbol{q}) \boldsymbol{\omega}_{0}\right]+\boldsymbol{\omega} \times \boldsymbol{J} \boldsymbol{\omega}
\end{aligned}
$$

\section{Simulation}

We assume that moment is $\boldsymbol{J}=\left[\begin{array}{ccc}800 & 0 & 0 \\ 0 & 800 & 0 \\ 0 & 0 & 600\end{array}\right] \mathrm{Kg} \cdot \mathrm{m}^{2}$, the orbit angular velocity is $\omega_{0}=0.001 \mathrm{rad} / \mathrm{s}$, the angular momentum of the SGCMG is10Nms, the maximum Euler angle velocity is $\dot{\phi}_{m}=2 \mathrm{deg} / \mathrm{s}$, the angular acceleration is $\ddot{\phi}_{m}=0.01 \mathrm{rad} / \mathrm{s}$, the initial attitude angle and attitude rate are $\boldsymbol{\theta}_{0}=\left[\begin{array}{lll}0 & 0 & 0\end{array}\right]^{T}$ deg and $\omega_{r 0}=\left[\begin{array}{lll}0 & 0 & 0\end{array}\right]^{T}$, while the target attitude angle and attitude rate are $\boldsymbol{\theta}_{c}=\left[\begin{array}{lll}40 & -20 & -10\end{array}\right]^{T}$ deg and $\omega_{r 0}=\left[\begin{array}{lll}0 & 0 & 0\end{array}\right]^{T}$.

The curves of attitude angles and attitude angular velocity show in figure 2 and the curves of triaxial output moment, Angular Momentum and singularity degree show in figure 3 . The simulation results show that: (1) the variable structure controller can accomplish the large angle attitude maneuver around the Euler axis according to the requirements; (2) During the attitude maneuver, the SGCMG system is always far away from the singular state. 

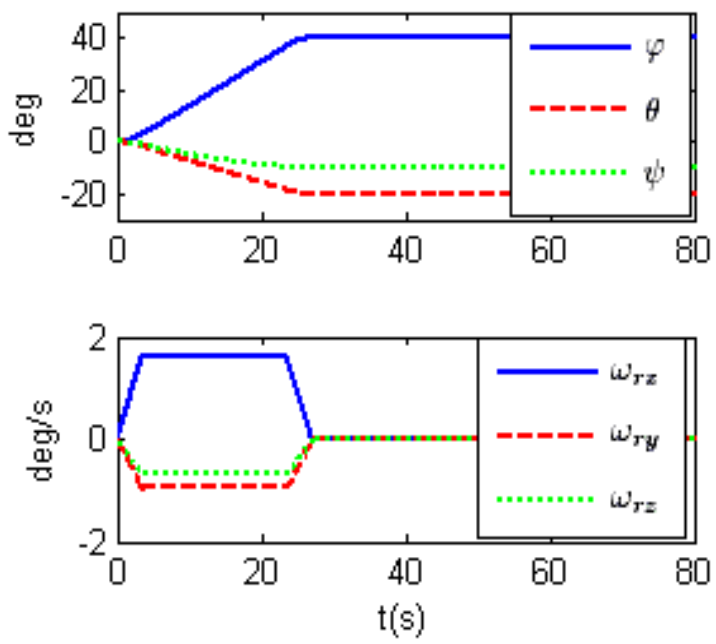

Figure 2. The curves of attitude angles and attitude angular velocity
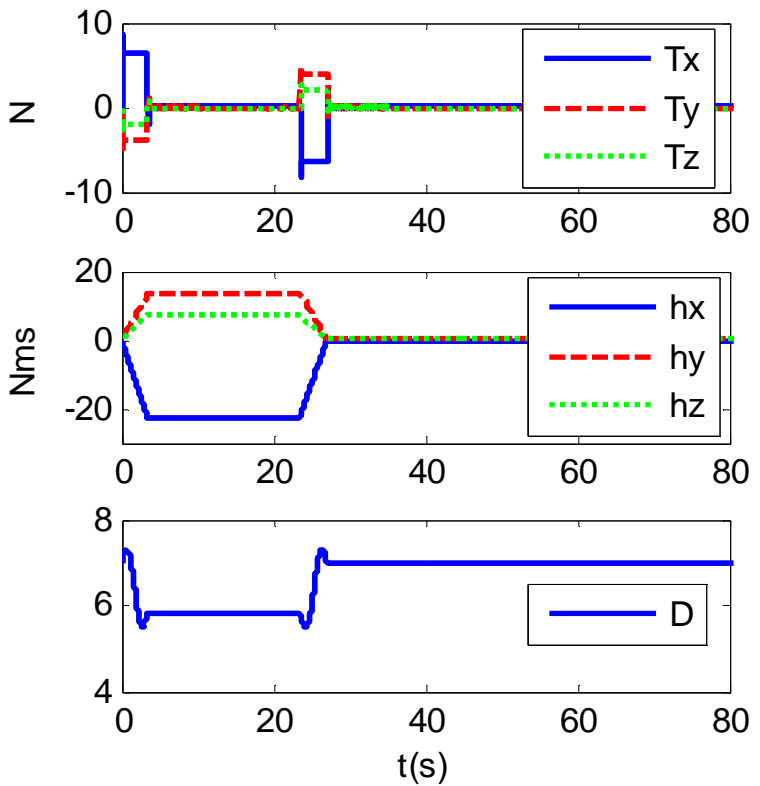

Figure 3. The curves of triaxial output moment, Angular Momentum and singularity degree

\section{Conclusion}

In order to realize the fast attitude maneuver of agile satellite, the angular trajectory of attitude maneuver around Euler axis is planned in this paper. To complete the planned attitude tracking, a variable structure control law based on error quaternion and error angular velocity is designed, and the Pentagon configuration composed of six SGCMGs is used as the attitude control system. The simulation results show that the control algorithm can effectively complete the attitude maneuver control tasks, and is insensitive to the changes of satellite parameters, showing strong robustness and good control performance.

\section{References}

[1] Wie B, Bailey D and Heiberg C. 2001. Singularity robust steering logic for redundant singlegimbal control moment gyros. Journal of Guidance, Control, and Dynamics, 24(5), pp865-872.

[2] Wie B. 2003. New singularity escape/avoidance steering logic for control moment gyro systems. AIAA Guidance, Navigation, and Control Conference and Exhibition. 
[3] Frederick A L and Norman G F. 2010. Hybrid steering logic for single-gimbal control moment gyroscopes. Journal of Guidance, Control, and Dynamics, 33(4), pp1201-1212.

[4] Jonathan W W, Eric D S and Frederick A L. 2012. Hardware testing of hybrid steering logic for single-gimbal control moment gyroscopes[C]. AIAA Guidance, Navigation, Control Conference.

[5] Guzman R, Luis G and Castilla M. 2018. Variable structure control in natural frame for threephase grid-connected inverters with LCL filter. IEEE Transactions on Power Electronics, 33(5), pp4512-4522 